\title{
Kisspeptin-10 inhibits the migration of breast cancer cells by regulating epithelial-mesenchymal transition
}

\author{
GUO-QING SONG and YI ZHAO \\ Department of Pancreas and Breast Surgery, Shengjing Hospital of China Medical University, \\ Heping, Shenyang, Liaoning 110004, P.R. China
}

Received September 3, 2014; Accepted October 26, 2014

DOI: $10.3892 /$ or.2014.3619

\begin{abstract}
Breast cancer is the leading cause of cancer-related death in women. Kisspeptin-10 (KP-10) is a shorter fragment of KISS1. In the present study, we demonstrated the antitumor effects of KP-10 on human breast cancer cell lines, MDA-MB231 and MDA-MB-157, both in vitro and in vivo. KP-10 was observed to induce apoptosis and inhibit the mobility of MDA-MB-231 and MDA-MB-157 cells. Correspondingly, KP-10 suppressed tumor growth in established xenograft tumor models and improved the survival rate of tumor-bearing mice. The formation of intratumoral microvessels was inhibited following treatment with KP-10. Finally, we confirmed that KP-10 inhibited cell mobility via epithelial-mesenchymal transition (EMT). Overall, the present study demonstrated that KP-10 suppressed breast cancer and human umbilical vein endothelial cell (HUVEC) growth both in vivo and in vitro. KP-10 is a novel regulator of EMT in breast cancer cells. However, additional studies are needed to confirm these results in other cell types.
\end{abstract}

\section{Introduction}

Breast cancer is the leading cause of cancer-related death in women and the third most common tumor worldwide (1). While significant treatment advances have been achieved, all breast cancer patients face the risk of metastasis to other organs, which is the main cause of death from breast cancer $(2,3)$. The etiology of breast cancer is a complex combination of both environmental and genetic factors; thus, the determination of genetic changes provides a new way to investigate the etiology of such a complex genetic disease (4).

The KISS1 gene was originally identified as a metastasis suppressor gene in human melanoma and breast carcinoma cells (5). The KISS1 gene encodes a 145-amino acid peptide that is cleaved into an amidated C-terminal 54-amino acid

Correspondence to: Dr Yi Zhao, Department of Pancreas and Breast Surgery, Shengjing Hospital of China Medical University, 36 Sanhao Street, Heping, Shenyang, Liaoning 110004, P.R. China E-mail: zhaozhaoyi_sj@163.com

Key words: KISS1, breast cancer, kisspeptin-10, apoptosis, mobility product, kisspeptin or metastin. Shorter fragments of kisspeptin-54, kisspeptin-14, kisspeptin-13, and kisspeptin-10 (KP-10), also bind to GPR54 (6,7). KISS1 transfection was found to lead to reduced invasion and migration of endometrial carcinoma (8) and breast cancer cells (9). Treatment with KP-10 showed similar effects in cancer cell lines including breast and endometrial carcinoma cells (10). However, the mechanisms of KP-10 in breast cancer cells remain unclear.

Epithelial-mesenchymal transition (EMT) is an essential developmental process through which cells of epithelial origin lose cell-cell contacts and cell polarity and acquire mesenchymal phenotypes (11). More and more evidence shows that EMT is the key means through which cancer cells acquire more highly mobile potentials to migrate and metastasize to distant sites during tumor progression (12).

In the present study, we sought to examine the association between KP-10 and EMT in human breast cancer cells. We demonstrated that KP-10 regulates EMT in breast cancer cells in vitro and in vivo. It is anticipated that the results of this study will provide novel insight into potential strategies for the treatment of breast cancer.

\section{Materials and methods}

Cell culture. MDA-MB-231 and MDA-MB-157 cell lines were purchased from the American Type Culture Collection (Rockville, MD, USA) and maintained in Dulbecco's modified Eagle's medium (DMEM) supplemented with $10 \%$ fetal calf serum (FCS) and antibiotics (100 $\mu \mathrm{M}$ penicillin and $100 \mu \mathrm{M}$ streptomycin). Human umbilical vein endothelial cells (HUVECs) were purchased from the Shanghai Institute of Biochemistry (Shanghai, China) and maintained in RPMI1640 medium supplemented with $10 \%$ FCS and antibiotics (100 $\mu \mathrm{M}$ penicillin and $100 \mu \mathrm{M}$ streptomycin). Cells were maintained in a humidified cell incubator with $5 \% \mathrm{CO}_{2}$ at $37^{\circ} \mathrm{C}$.

MTT assay. Viability of cells was determined using the 3-(4,5-dimethylthiazolyl-2)-2,5-diphenyltetrazolium bromide (MTT) assay (Sigma-Aldrich, Carlsbad, CA, USA). MDA-MB231 cells, MDA-MB-157 cells or HUVECs were plated at a density of $5 \times 10^{3}$ cells $/ \mathrm{cm}^{2}$, cultured until $60 \%$ confluency was reached and treated with various concentrations of KP-10 (Tyr-Asn-Trp-Asn-Ser-Phe-Gly-Leu-Arg-Phe) (ChinaPeptides 
Co., Ltd., Biomart.cn, Shanghai, China) $(0,20,40,60,80$ and $100 \mathrm{ng}$ ) for $48 \mathrm{~h}$. The cells were treated with $0.5 \mathrm{mg} / \mathrm{ml}$ MTT for $4 \mathrm{~h}$ and lysed with dimethyl sulfoxide (DMSO). Absorbance rates were measured at $550-560 \mathrm{~nm}$ using a microplate reader (Bio-Rad, Hercules, CA, USA).

Cell apoptosis assay. Apoptosis was determined using an apoptosis detection kit (KeyGen, Nanjing, China). Briefly, cells were collected, washed twice in ice-cold phosphate-buffered saline (PBS), and then resuspended in binding buffer at a density of $1 \times 10^{6}$ cells $/ \mathrm{ml}$. Cells were simultaneously incubated with fluorescein-labeled Annexin V and propidium iodide (PI) for $20 \mathrm{~min}$. The mixture was then analyzed on a FACSCalibur (BD Biosciences, Baltimore, MD, USA). Annexin V-FITC generated signals were detected on FL1 at a wavelength of $525 \mathrm{~nm}$, and PI signals were monitored using a detector reserved for phycoerythrin emission (FL2, $575 \mathrm{~nm}$ ). Data were analyzed using CellQuest software from BD.

Transwell migration assay. The migration assay was performed using a Boyden chamber $(8-\mu \mathrm{m}$ pore size polycarbonate membrane; Cell Biolabs, San Diego, CA, USA). Cells were resuspended in FBS-free DMEM to a concentration of $3 \times 10^{5}$ cells $/ \mathrm{ml}$. The upper chamber was loaded with $200 \mu 1$ of cell suspension and the lower chamber was loaded with $600 \mu \mathrm{l}$ of DMEM containing 10\% FBS. The filter was fixed in $4 \%$ paraformaldehyde (Sigma) and stained with crystal violet (CV; Beyotime, Shanghai, China). The cells on the upper side of the filter were wiped off using a cotton swab. The cells that migrated to the undersurface of the membrane were counted using a light microscope. Ten microscopic fields $(x 400)$ were randomly selected to count the cells.

In vitro wound healing assay. Cells were grown in a 6-well dish. A confluent monolayer of cells was scratched with a 200- $\mu$ l pipette tip to simulate a wound. Cells were washed twice with PBS and then supplemented with medium and incubated for $4 \mathrm{~h}$ at $37^{\circ} \mathrm{C}$. Cell migration into the wounded area was monitored microscopically. Images were captured at the interface of the unwounded and wounded areas.

FLIPR Calcium 4 assay. Cell monolayers were plated in black, clear-bottomed 96-well plates overnight. Cells were then incubated in serum-free media at $37^{\circ} \mathrm{C}$ for $1 \mathrm{~h}$ prior to stimulation with FLIPR Calcium 4 reagent (Molecular Devices, Sunnyvale, CA, USA). Cells were then incubated for $1 \mathrm{~h}$ at $37^{\circ} \mathrm{C}$ while ligands were prepared in a separate flat-bottomed 96-well plates. Cells were then tested for intensity of fluorescence on the NOVOstar (BMG Labtech, UK) over $60 \mathrm{sec}$ with ligands being added 1:10 at $11 \mathrm{sec}$.

Gelatin zymography. Fifty micrograms of protein was applied to $10 \%$ polyacrylamide gels with $1 \%$ gelatin incorporated as a substrate for gelatinolytic proteases. After running the gel the SDS was removed by washing twice in $2.5 \%$ Triton X-100 for $30 \mathrm{~min}$. The gels were incubated overnight in zymography development buffer containing $50 \mathrm{mM}$ Tris- $\mathrm{HCl}(\mathrm{pH}$ 7.4), $2 \mathrm{mM} \mathrm{NaN}_{3}$ and $5 \mathrm{mM} \mathrm{CaCl}_{2}$. After development, the gels were stained for $3 \mathrm{~h}$ in $45 \%$ methanol/10\% glacial acetic acid containing $1 \%(\mathrm{w} / \mathrm{v})$ Coomassie blue R-250 and subsequently partially destained with the same solution without dye. The gelatinolytic activity of each MMP was evaluated as a clear band against the blue-stained gelatin background.

Animals. The present study was conducted using protocols approved by the Animal Care and Use Committee of China Medical University. NOD/SCID mice were allowed a standard rat diet and water ad libitum, and maintained on a 10-/14-h light/dark cycle. MDA-MB-231 or MDA-MB-157 cells $\left(1 \times 10^{8}\right)$ were injected into NOD/SCID mice by subcutaneous injection. After the tumor diameters reached 3-5 mm, the mice were divided randomly into 6 groups (MDA-MB-231, MDA-MB-231 + PBS, MDA-MB-231 + KP-10, MDA-MB-157, MDA-MB-157 + PBS and MDA-MB-157 + KP-10). Tumor growth was monitored every 5 days after the first week of inoculation. All mice were sacrificed at day 60. All of the tumor tissues were collected, fixed in $4 \%$ formaldehyde and embedded in paraffin for immunostaining.

Immunostaining. For immunohistochemical staining of CD31, endogenous peroxidase activity was blocked in $4-\mu \mathrm{m}$ tumor sections with $3 \%$ hydrogen peroxide for $30 \mathrm{~min}$. Antigen retrieval was performed in citrate buffer $(10 \mathrm{mM}, \mathrm{pH}$ 6.0) for $30 \mathrm{~min}$ at $95^{\circ} \mathrm{C}$ in a pressure cooker. The CD31 antibody (Sigma) was incubated with the sections at 1:500 overnight at $4^{\circ} \mathrm{C}$. The sections were then incubated with a biotinylated secondary antibody for $1 \mathrm{~h}$ at room temperature, followed by incubation with a streptavidin-horseradish peroxidase (HRP) complex (Beyotime, Beijing, China) for $60 \mathrm{~min}$ at room temperature. The bound antibody was visualized with 3,3'-diaminobenzidine tetrahydrochloride (DAB; Beyotime). Sections were also counterstained with hematoxylin (Beyotime).

Chick embryo chorioallantoic membrane (CAM) angiogenesis assay. According to the method of Hong et al (13), the fertilized eggs were incubated at $37^{\circ} \mathrm{C}$ for 8 days and then divided into 2 groups (PBS and KP-10). After incubation for 3 more days, the CAM of each live egg was harvested and placed individually in a 6-well plate. Images of the blood vessels of each CAM were captured using Motic Images Plus ${ }^{\circledR}$ (MediaCybernetics ${ }^{\circledR}$, Bethesda, MD, USA). The blood vessel area as a percentage of the total CAM area, blood vessel length and number of branch points were quantified using Image Pro ${ }^{\circledR}$ analysis software (MediaCybernetics ${ }^{\circledR}$ ).

Western blot analysis. Proteins were resolved by $10 \%$ SDS-PAGE, transferred to a nitrocellulose membrane, and detected using the following antibodies: E-cadherin (sc-7870), vimentin (sc-6260), N-cadherin (sc-7939) and $\beta$-actin (sc-47778) (all from Santa Cruz Biotechnology, Santa Cruz, CA, USA). Immunostaining was detected using an enhanced chemiluminescence (ECL) system (Amersham Biosciences, Westborough, MA, USA).

Statistical analysis. Data are presented as the means \pm standard deviation (SD). Differences between groups were analyzed using the Student's t-test for continuous variables. The Kaplan-Meier estimator was used to compare different patient groups, and P-values were calculated using the log-rank (Mantel-Cox) test. Statistical analysis was performed using 

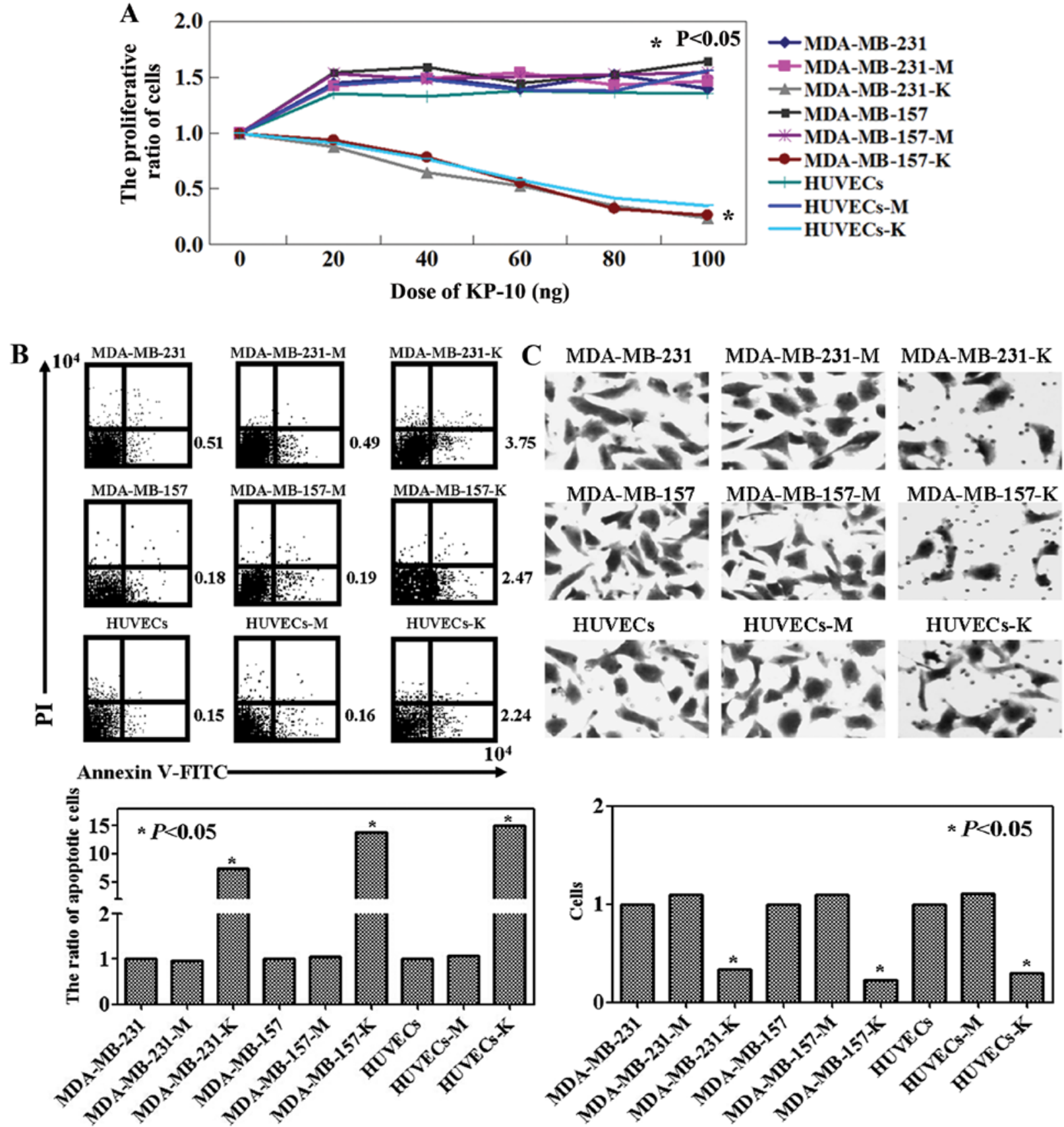

Figure 1. Antitumor activities of KP-10 in vitro. (A) Growth curves of MDA-MB-231 cells, MDA-MB-157 cells and HUVECs treated with different concentrations of KP-10. (B) The proportion of apoptotic cells was determined by double-staining with Annexin V/FITC and PI. (C) The mobility of the cells was determined using a Transwell assay. MDA-MB-231, untreated MDA-MB-231 cells; MDA-MB-231-M, MDA-MB-231 cells treated with PBS; MDAMB-231-K, MDA-MB-231 cells treated with KP-10; MDA-MB-157, untreated MDA-MB-157 cells; MDA-MB-157-M, MDA-MB-157 cells treated with PBS MDA-MB-157-K, MDA-MB-157 cells treated with KP-10; HUVECs, untreated HUVECs; HUVECs-M, HUVECs treated with PBS; HUVECs-K, HUVECs treated with KP-10. KP-10, kisspeptin-10; HUVECs, human umbilical vein endothelial cells.

GraphPad 5.0 software (GraphPad Software, La Jolla, CA, USA) and significance was established at $\mathrm{P}<0.05$.

\section{Results}

Effects of KP-10 on breast cancer cells in vitro. The inhibitory growth effects of KP-10 on breast cancer MDA-MB-231 and MDA-MB-157 cells were determined by MTT assay. This inhibition was dependent on the concentration of KP-10. The $\mathrm{IC}_{50}$ values of KP-10 for MDA-MB-231 and MDA-MB-157 cells were 62.5 and $63.3 \mathrm{nM}$, respectively ( $\mathrm{P}<0.05$; Fig. 1A). The apoptotic ratio of KP-10-treated MDA-MB-231 or MDA-MB157 cells was 5-15 times higher compared with the ratio of the respective control cells $(\mathrm{P}<0.05$; Fig. $1 \mathrm{~B})$. The mobility of breast cancer cells was also inhibited by KP-10. The Transwell assay showed that less cells after KP-10 treatment migrated to the lower side of the membrane than the number of cells in the untreated ones $(\mathrm{P}<0.05$; Fig. $1 \mathrm{C})$. The wound-healing assay showed that the migration of MDA-MB-231 or MDA-MB-157 cells after KP-10 treatment was significant reduced when 

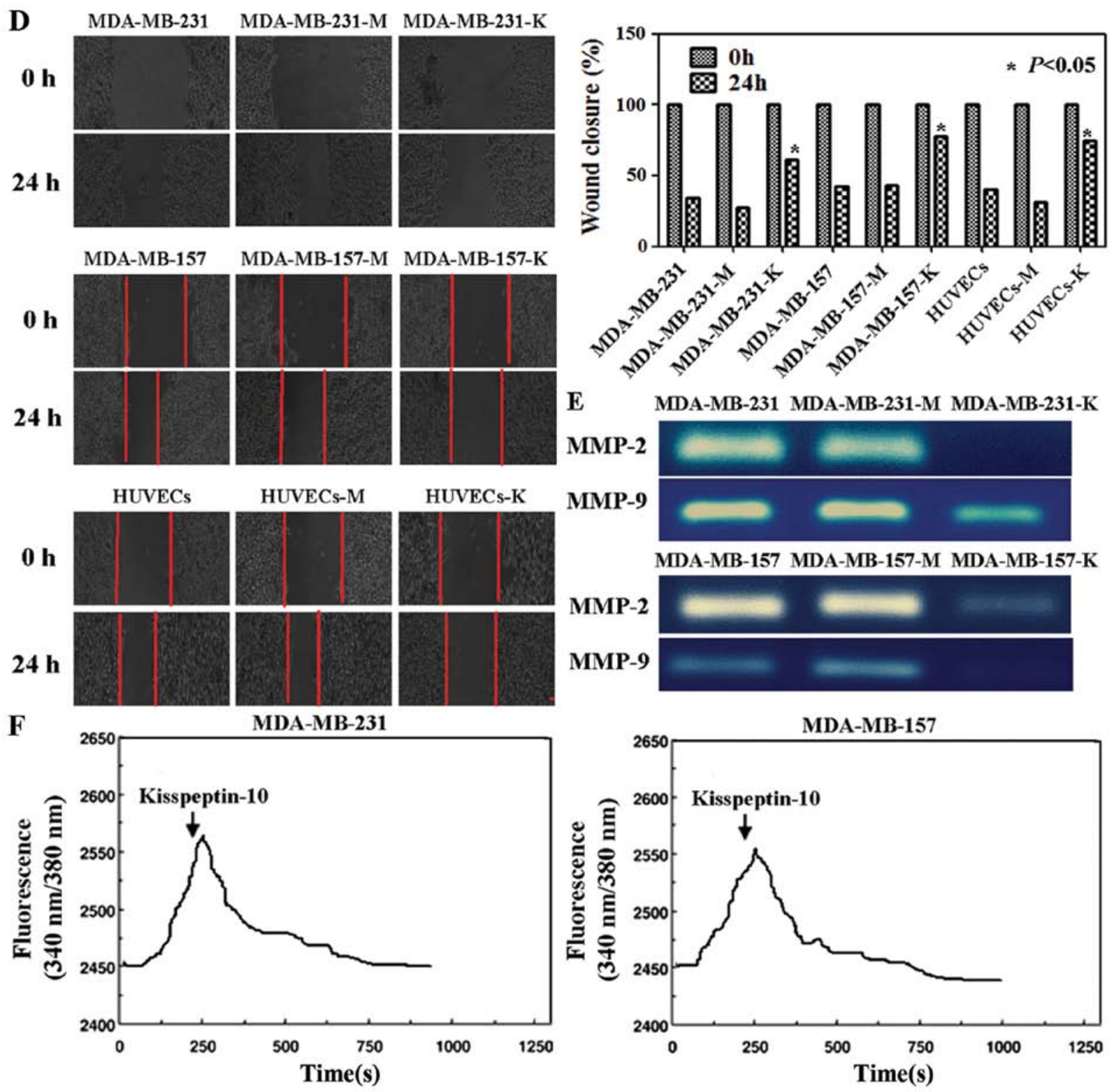

Figure 1. Continued. (D) Wound closure was quantified in the regions flanked by dotted lines. (E) Gelatinolytic activity of secreted MMP-2 and MMP-9 in the breast cancer cells was analyzed by zymography. (F) The concentration of $\mathrm{Ca}^{2+}$ was measured using FLIPR Calcium 4 assay. MDA-MB-231, untreated MDA-MB-231 cells; MDA-MB-231-M, MDA-MB-231 cells treated with PBS; MDA-MB-231-K, MDA-MB-231 cells treated with KP-10; MDA-MB-157, untreated MDA-MB-157 cells; MDA-MB-157-M, MDA-MB-157 cells treated with PBS; MDA-MB-157-K, MDA-MB-157 cells treated with KP-10; HUVECs, untreated HUVECs; HUVECs-M, HUVECs treated with PBS; HUVECs-K, HUVECs treated with KP-10. KP-10, kisspeptin-10; HUVECs, human umbilical vein endothelial cells.

compared with that in the untreated cells $(\mathrm{P}<0.05$; Fig. 1D). We also found that the activity of MMP-2 and MMP-9 was inhibited by KP-10 in the MDA-MB-231 and MDA-MB157 cells (Fig. 1E). Furthermore, both MDA-MB-231 and MDA-MB-157 cells exhibited a marked increase in $\left[\mathrm{Ca}^{2+}\right]$ in response to treatment with KP-10 (Fig. 1F). Notably, KP-10 had similar effects on HUVECs (Fig. 1).

Antitumor activity of KP-10 in a breast cancer mouse model. The antitumor properties of KP-10 were evaluated using mouse breast cancer models. Both tumor volume and the weight of the KP-10-treated tumors were lower compared to the untreated ones $(\mathrm{P}<0.05$; Fig. $2 \mathrm{~A}$ and $\mathrm{B})$. KP-10 also increased the survival rate of the tumor-bearing mice $(\mathrm{P}<0.05$; Fig. $2 \mathrm{C})$.
In addition, the blood vessel density of the tumors from the mice in the KP-10-treated group was significantly reduced compared to that in the untreated group using immunostaining for $\mathrm{CD} 31$ expression ( $\mathrm{P}<0.05$; Fig. 2D).

We evaluated the inhibitory effect of KP-10 on in vivo angiogenesis by using a chick embryo chorioallantoic membrane angiogenesis assay. The results showed that KP-10 inhibited neovascularization in the chick CAM model $(\mathrm{P}<0.05$; Fig. 2E).

KP-10 induces EMT in breast cancer cells. Based on the antitumor roles of KP-10 in breast cancer cells, we carried out western blotting to identify the mechanism of KP-10. Compared with the untreated cells, KP-10-treated MDA-MB-231 and 
$\mathbf{A}$

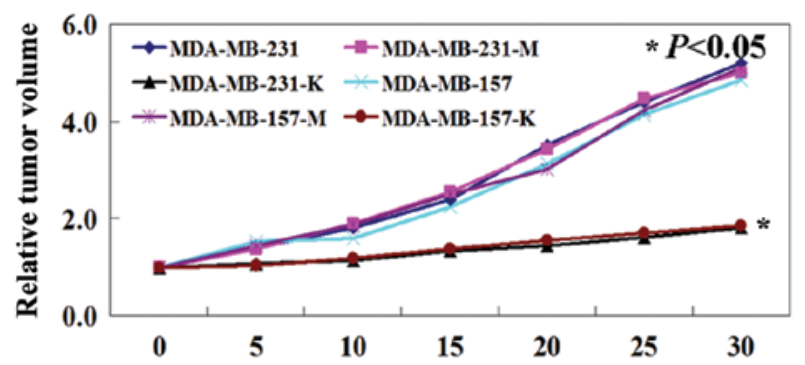

Time after treatment (days)

B

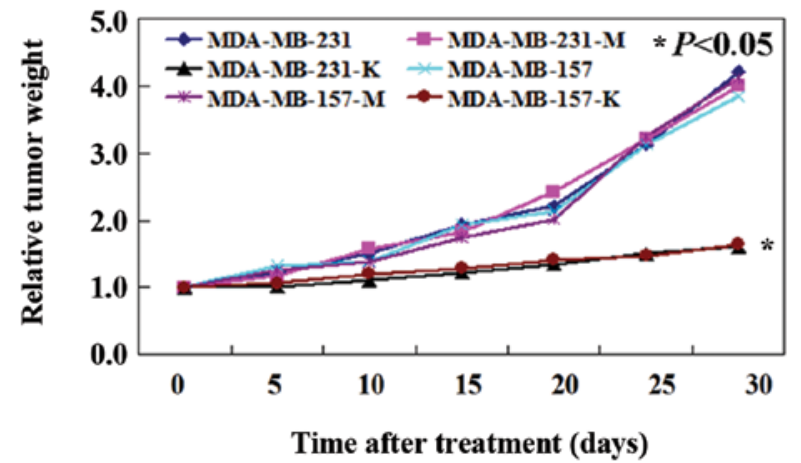

C

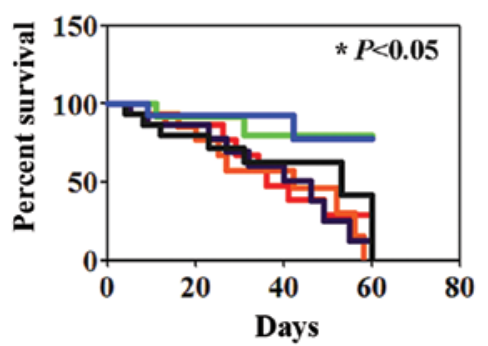

D MDA-MB-231 MDA-MB-231-M MDA-MB-231-K

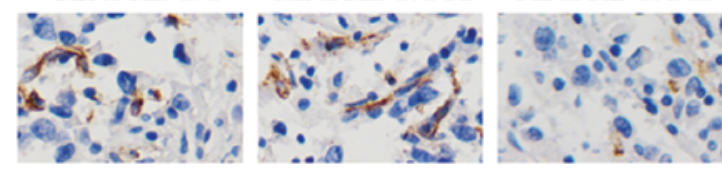

MDA-MB-157 MDA-MB-157-M MDA-MB-157-K
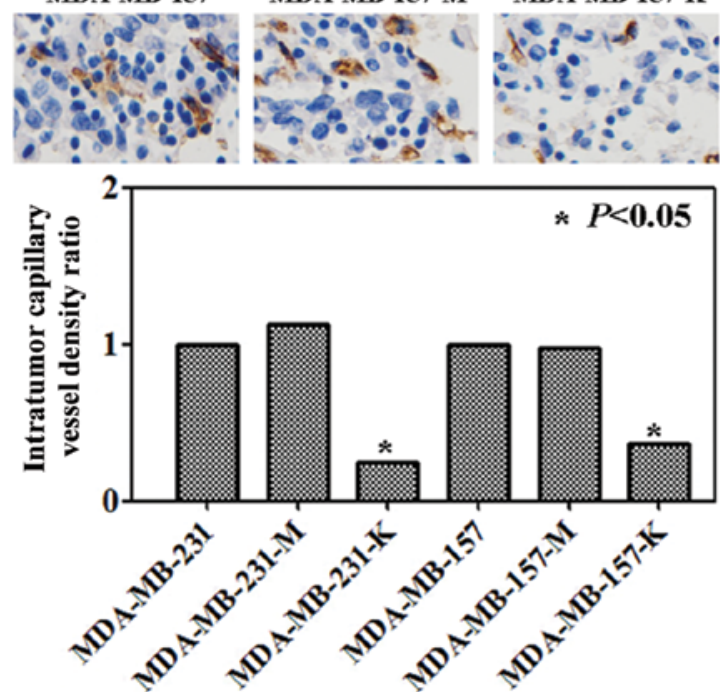

E Untreated PBS
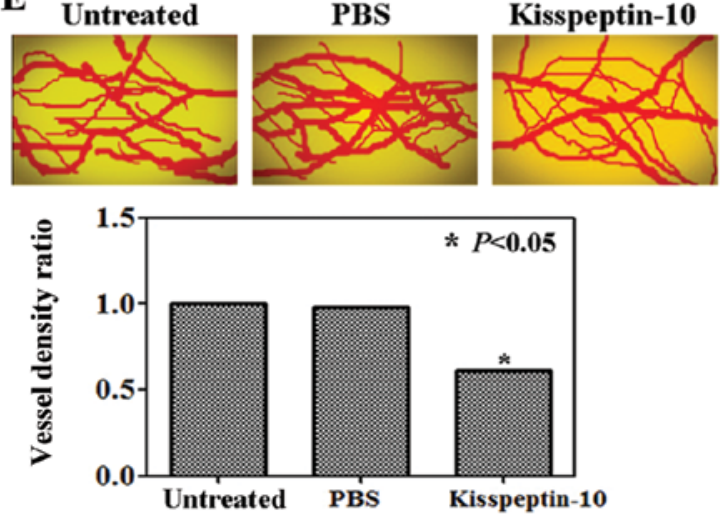

Figure 2. KP-10 suppresses tumor growth in xenograft mouse models. (A and B) Tumor volume and tumor weight of each mouse group as described in Materials and methods. Mice were sacrificed 20 days after the cells were injected. (C) Kaplan-Meier survival curves of the mouse groups described in Materials and methods. (D) Immunohistochemical staining of tumor vessel endothelial cells using an anti-CD31 antibody. (E) KP-10 inhibits VEGF-induced angiogenesis in vivo in the chick embryo model. KP-10, kisspeptin-10. MDA-MB-231, untreated MDA-MB-231 cells; MDA-MB-231-M, MDA-MB-231 cells treated with PBS; MDA-MB-231-K, MDA-MB-231 cells treated with KP-10; MDA-MB-157, untreated MDA-MB-157 cells; MDA-MB-157-M, MDA-MB-157 cells treated with PBS; MDA-MB-157-K, MDA-MB-157 cells treated with KP-10.
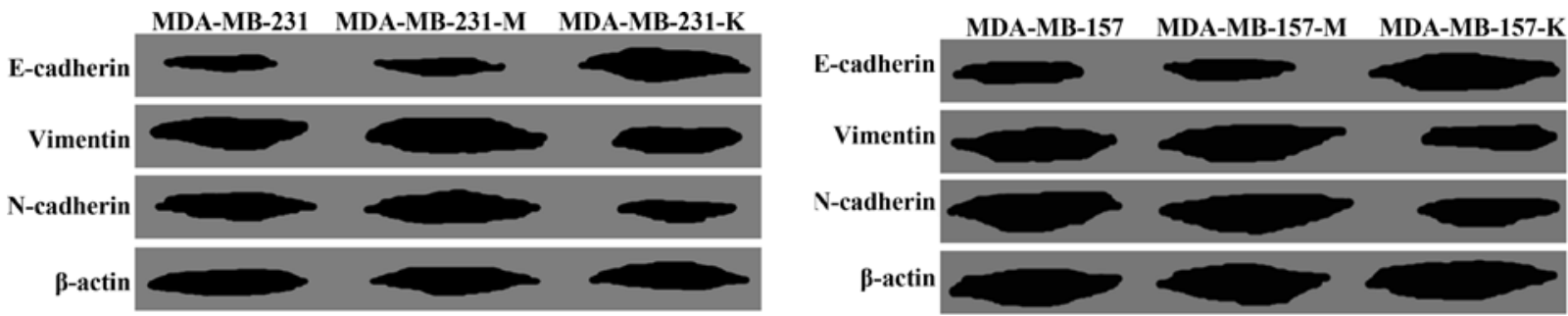

Figure 3. Western blot analysis was performed using E-cadherin, N-cadherin and vimentin. KP-10 was significantly associated with high expression of E-cadherin, yet adversely with low N-cadherin expression. MDA-MB-231, untreated MDA-MB-231 cells; MDA-MB-231-M, MDA-MB-231 cells treated with PBS; MDA-MB-231-K, MDA-MB-231 cells treated with KP-10; MDA-MB-157, untreated MDA-MB-157 cells; MDA-MB-157-M, MDA-MB-157 cells treated with PBS; MDA-MB-157-K, MDA-MB-157 cells treated with KP-10. KP-10, kisspeptin-10.

MDA-MB-157 cells showed a higher expression level of epithelial marker E-cadherin, while lower expression levels of mesenchymal markers vimentin and $\mathrm{N}$-cadherin were noted (Fig. 3). 


\section{Discussion}

In the present study, we found that EMT is involved in the inhibition of migration in breast cancer cells caused by KP-10. The metastasis suppressor activity of KPs has been identified in numerous types of cancers, including brain (14), gastric (15), bladder (16) and ovarian (17). Consistent with previous studies, we also noted a metastasis suppressor role of KP-10 in breast cancer cells. Some studies have shown that KP-induced metastatic suppression is related with the suppression of matrix metalloproteinase (MMP)-9 activity $(5,18,19)$. Our finding showed that KP-10 increased MMP-9 and MMP-2 activity and subsequently inhibited cancer cell migration and invasion. A significant feature of MDA-MB-231 and MDA-MB-157 cell lines is that KP-10 can stimulate intracellular calcium release. Roseweir et al (20) found that a similar effect of KP-10 could induce $\mathrm{Ca}^{2+}$ mobilization in extravillious trophoblast-derived cells, HTR8SV. However, they did not find IP3 release during this process. We will investigate the mechanism of $\mathrm{KP}-10$-induced $\mathrm{Ca}^{2+}$ release in future studies. KP has been shown to inhibit angiogenesis in tumors and the placenta (21). Cho et al (22) found that KP-10 inhibited the migration and invasion of HUVECs. Usui et al (23) also found that KP-10 inhibited neovascularization in ischaemic limb muscle and inhibited the proliferation of HUVECs. In the present study, we confirmed that KP-10 inhibited the migration of breast cancer cells and HUVECs. Furthermore, we used chick embryo chorioallantoic membrane angiogenesis assay to confirm the angiogenesis inhibitory role of KP-10 in vivo.

EMT is a process by which epithelial cells change to a mesenchymal phenotype and is a crucial step in the initiation of the metastatic spread of many tumor cells into distal organs (24). EMT is characterized by a switch from E-cadherin to N-cadherin expression (25). The loss or reduction in E-cadherin expression is a key event in the initiation of EMT (26). We here showed that KP-10 was significantly associated with high expression of E-cadherin, yet adversely with low $\mathrm{N}$-cadherin expression.

Collectively, the present study revealed that KP-10 suppressed breast cancer and endothelial cell growth both in vivo and in vitro. KP-10 is a novel regulator of EMT and metastasis in breast cancer cells.

\section{Acknowledgements}

We are indebted to Su-Ning Zhang for his helpful criticism of the manuscript and excellent technical assistance.

\section{References}

1. Jemal A, Bray F, Center MM, et al: Global cancer statistics. CA Cancer J Clin 61: 69-90, 2011.

2. Culhane AC and Quackenbush J: Confounding effects in 'a six-gene signature predicting breast cancer lung metastasis'. Cancer Res 69: 7480-7485, 2009.

3. Chiang AC and Massagué J: Molecular basis of metastasis. N Engl J Med 359: 2814-2823, 2008.

4. Theodoropoulos GE, Saridakis V, Karantanos T, et al: Toll-like receptors gene polymorphisms may confer increased susceptibility to breast cancer development. Breast 21: 534-538, 2012.
5. Makri A, Pissimissis N, Lembessis $\mathrm{P}$, et al: The kisspeptin (KiSS-1)/GPR54 system in cancer biology. Cancer Treat Rev 34: 682-692, 2008.

6. Muir AI, Chamberlain L, Elshourbagy NA, et al: AXOR12, a novel human $\mathrm{G}$ protein-coupled receptor, activated by the peptide KiSS-1. J Biol Chem 276: 28969-28975, 2001.

7. Kotani M, Detheux M, Vandenbogaerde A, et al: The metastasis suppressor gene KiSS-1 encodes kisspeptins, the natural ligands of the orphan $\mathrm{G}$ protein-coupled receptor GPR54. J Biol Chem 276: 34631-34636, 2001.

8. Jiang T, Zhang SL, Lin B, et al: Expression and clinical significance of KiSS-1 and GPR54 mRNA in endometrial carcinoma. Zhonghua Zhong Liu Za Zhi 27: 229-231, 2005 (In Chinese).

9. Song GQ and Zhao Y: Different therapeutic effects of distinct KISS1 fragments on breast cancer in vitro and in vivo. Int J Oncol 43: 1219-1227, 2013.

10. Olbrich T, Ziegler E, Türk G, et al: Kisspeptin-10 inhibits bone-directed migration of GPR54-positive breast cancer cells: evidence for a dose-window effect. Gynecol Oncol 119: 571-578, 2010.

11. Lim $\mathbf{J}$ and Thiery JP: Epithelial-mesenchymal transitions: insights from development. Development 139: 3471-3486, 2012.

12. Scheel C and Weinberg RA: Phenotypic plasticity and epithelialmesenchymal transitions in cancer and normal stem cells? Int J Cancer 129: 2310-2314, 2011.

13. Hong J, Zhang Z, Lv W, et al: Icaritin synergistically enhances the radiosensitivity of $4 \mathrm{~T} 1$ breast cancer cells. PLoS One 8: e71347, 2013.

14. Zohrabian VM, Nandu H, Gulati N, et al: Gene expression profiling of metastatic brain cancer. Oncol Rep 18: 321-328, 2007.

15. Dhar DK, Naora H, Kubota H, et al: Downregulation of KiSS-1 expression is responsible for tumor invasion and worse prognosis in gastric carcinoma. Int J Cancer 111: 868-872, 2004.

16. Cebrian V, Fierro M, Orenes-Piñero E, et al: KISS1 methylation and expression as tumor stratification biomarkers and clinical outcome prognosticators for bladder cancer patients. Am J Pathol 179: 540-546, 2011.

17. Hata K, Dhar DK, Watanabe Y, Nakai H and Hoshiai H: Expression of metastin and a G-protein-coupled receptor (AXOR12) in epithelial ovarian cancer. Eur J Cancer 43: 1452-1459, 2007.

18. Li N, Wang HX, Zhang J, Ye YP and He GY: KISS-1 inhibits the proliferation and invasion of gastric carcinoma cells. World $\mathrm{J}$ Gastroenterol 18: 1827-1833, 2012

19. Navenot JM, Fujii N and Peiper SC: Activation of Rho and Rho-associated kinase by GPR54 and KiSS1 metastasis suppressor gene product induces changes of cell morphology and contributes to apoptosis. Mol Pharmacol 75: 1300-1306, 2009.

20. Roseweir AK, Katz AA and Millar RP: Kisspeptin-10 inhibits cell migration in vitro via a receptor-GSK3 beta-FAK feedback loop in HTR8SVneo cells. Placenta 33: 408-415, 2012.

21. Ramaesh T, Logie JJ, Roseweir AK, et al: Kisspeptin-10 inhibits angiogenesis in human placental vessels ex vivo and endothelial cells in vitro. Endocrinology 151: 5927-5934, 2010.

22. Cho SG, Yi Z, Pang X, et al: Kisspeptin-10, a KISS1-derived decapeptide, inhibits tumor angiogenesis by suppressing Sp-1mediated VEGF expression and FAK/Rho GTPase activation. Cancer Res 69: 7062-7070, 2009.

23. Usui S, Iso Y, Sasai M, et al: Kisspeptin-10 induces endothelial cellular senescence and impaired endothelial cell growth. Clin Sci 127: 47-55, 2014.

24. Thiery JP, Acloque H, Huang RY and Nieto MA: Epithelialmesenchymal transitions in development and disease. Cell 139: $871-890,2009$

25. Nakajima S, Doi R, Toyoda E, et al: N-cadherin expression and epithelial-mesenchymal transition in pancreatic carcinoma. Clin Cancer Res 10: 4125-4133, 2004.

26. Werling AM, Doerflinger Y, Brandner JM, et al: Homo- and heterotypic cell-cell contacts in Merkel cells and Merkel cell carcinomas: heterogeneity and indications for cadherin switching. Histopathology 58: 286-303, 2011. 